First radiation hardness results of the TeraPixel Active Calorimeter (TPAC) sensor

This content has been downloaded from IOPscience. Please scroll down to see the full text. 2013 JINST 8 P01007

(http://iopscience.iop.org/1748-0221/8/01/P01007)

View the table of contents for this issue, or go to the journal homepage for more

Download details:

IP Address: 137.138.125.163

This content was downloaded on 08/07/2014 at 07:50

Please note that terms and conditions apply. 


\section{First radiation hardness results of the TeraPixel Active Calorimeter (TPAC) sensor}

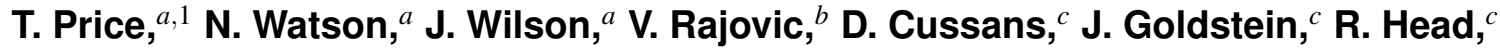
S. Nash, ${ }^{c}$ R. Page, ${ }^{c}$ J. Velthuis, ${ }^{c}$ J. Strube, ${ }^{d}$ M. Stanitzki, ${ }^{e}$ P. Dauncey, ${ }^{f}$ R. Gao, ${ }^{g}$ A. Nomerotski, ${ }^{g}$ R. Coath, ${ }^{h}$ J. Crooks, ${ }^{h}$ R. Turchetta, ${ }^{h}$ M. Tyndel,${ }^{h}$ S. Worm ${ }^{h}$ and Z. Zhang ${ }^{h}$

${ }^{a}$ Particle Physics Group, School of Physics and Astronomy, University of Birmingham, Edgbaston, B15 2TT, United Kingdom

${ }^{b}$ School of Electrical Engineering, University of Belgrade,

73 Bulevar Kralja Aleksandra, Belgrade, Serbia

${ }^{c}$ H.H. Wills Physics Laboratory, University of Bristol,

Tyndall Avenue, Bristol, BS8 1TL, United Kingdom

${ }^{d}$ CERN,

1211 Geneve 23, Switzerland

${ }^{e} \mathrm{DESY}$,

Notkestrasse 85, 22607, Hamburg, Germany

${ }^{f}$ Blackett Laboratory, Imperial College London,

Prince Consort Road, London, SW7 2AZ, United Kingdom

${ }^{g}$ Physics Department, University of Oxford,

Denys Wilkinson Building, Keble Road, Oxford, OX1 3RH, United Kingdom

${ }^{h}$ STFC Rutherford Appleton Laboratory,

Chilton, Didcot, OX11 0QX, United Kingdom

E-mail: txp@hep.ph.bham.ac.uk

Abstract: The TeraPixel Active Calorimeter (TPAC) sensor is a novel Monolithic Active Pixel Sensors (MAPS) device developed for use as the active layers of a large area, digital electromagnetic calorimeter (DECAL) at a future $e^{+} e^{-}$collider. Further applications, which include the tracking and vertex systems for future lepton colliders and LHC upgrades have been proposed and it is therefore essential to characterise the behaviour of the sensor for these applications. We present the first studies of radiation hardness testing of the TPAC sensor. The performance of the sensor has been evaluated after exposures up to $5 \mathrm{Mrad}$ of $50 \mathrm{keV}$ x-rays. Under realistic ILC operating conditions a maximum decrease in the signal to noise ratio of $8 \%(15 \%)$ was observed after $200 \mathrm{krad}$ (5 Mrad) which is already sufficient for proposed applications in future $e^{+} e^{-}$colliders.

KEYWORDS: Solid state detectors; Radiation damage to detector materials (solid state); Calorimeter methods

\footnotetext{
${ }^{1}$ Corresponding author.
} 


\section{Contents}

1 Introduction 1

2 Pixel technology 2

2.1 TPAC sensor 2

2.2 INMAPS process 3

3 Sensor characterisation 3

4 Irradiation studies $\quad 5$

4.1 Noise and pedestals — bulk pixels 5

4.1.1 Unpowered sensors

$\begin{array}{lll}\text { 4.1.2 Powered sensors } & 6\end{array}$

4.1.3 Shift register problems $\quad 8$

$\begin{array}{lll}4.2 & \text { Signal-analogue test pixels } & 10\end{array}$

4.3 Signal to noise 11

5 Conclusions 14

\section{Introduction}

The detection of minimum ionising particles (MIPs) has been successfully demonstrated using CMOS Monolithic Active Pixel Sensors (MAPS) [1]. This technology allows the development of low noise, high sensitivity, radiation tolerant sensors [2, 3]. The cost per unit area of standard CMOS sensors is expected to be considerably lower than other technologies making it possible to build a whole Electromagnetic Calorimeter (ECAL) with silicon readout. Sensor fabrication would be more straightforward, with integrated electronics and a much wider choice of vendors leading to increased production speed and capacity. The CALICE-UK Collaboration [4] developed the TeraPixel Active Calorimeter (TPAC) sensor for use in a binary readout (Digital) Electromagnetic Calorimeter (DECAL) within the detectors at a future $e^{+} e^{-}$collider such as the International Linear Collider (ILC). TPAC is the first prototype device for such an application. To date, no effort has been made to minimise the power consumption of the sensors, a characteristic which will be essential in a DECAL. Although the readout is based on ILC bunch structures (5Hz bunch trains, each consisting of $\sim 3000$ bunches separated by a few hundred ns) the timings could be optimised for alternative machines e.g. the Compact Linear Collider (CLIC) [5, 6].

Previous studies have demonstrated a wide tolerance of CMOS sensors to radiation from sensors able to withstand greater than $1 \mathrm{Mrad}$ [7] to sensors that can withstand a just a few krad [8,9]. The TPAC sensor was primarily designed to be used in the low radiation environment of an ECAL at the ILC and as such, creating a radiation hard sensor was not one of the key design features. As 


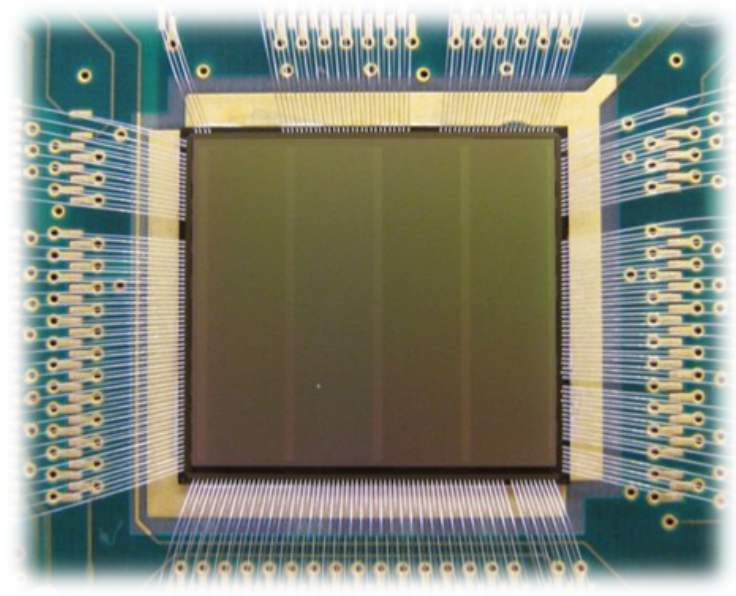

Figure 1. Photograph of a bonded TPAC sensor showing the four vertical regions and the columns of logic and SRAM (lighter regions) which serve them.

possible uses for the TPAC sensors and the INMAPS technology [10] have broadened, such as vertexing at the proposed SuperB Collider [11], tracking systems for the ALICE upgrade at LHC [12], and the higher radiation environment of the ECAL at CLIC, the sensors' response to radiation has been studied to characterise their performance.

Section 2 gives a brief overview of the TPAC sensor to be tested and the innovative INMAPS process which enables the use of CMOS sensors for efficient MIP detection. Section 3 outlines the methods used to characterise the pixels, allowing a quantitative evaluation of the impact of the irradiations. Finally, the results of the irradiations are presented in section 4 with particular emphasis on the noise and pedestal shifts, and the impact on the signal to noise ratio, and conclusions are given in section 5 .

\section{Pixel technology}

\subsection{TPAC sensor}

The TPAC sensor is the first sensor fabricated that utilises INMAPS technology (see section 2.2) and the benefits to charge collection and pixel efficiency have been successfully demonstrated [13, 14]. Due to the shower densities expected within the core of electromagnetic showers in a DECAL at a linear collider a pixel size of $50 \times 50 \mu \mathrm{m}^{2}$ was chosen for prototyping. This is required to ensure single pixel occupancy in events with a peak density of 100 particles $/ \mathrm{mm}^{2}$. The detector geometry thus leads to $\sim 10^{12}$ pixels in a complete DECAL system and the integrated readout electronics of the TPAC sensor are essential to minimise the dead space associated with each pixel.

The prototype sensor contains 28224 pixels in a $168 \times 168$ grid and is segmented in to four regions with each region served by a column of logic and SRAM for readout purposes (lighter, vertical areas in figure 1). Each region of the sensor is subdivided into 168 rows of 42 pixels with each row further divided into seven segments of six pixels [15]. Each pixel has four signal diodes and a hit is registered when the combined signal in a pixel goes above a given threshold (set in arbitrary units of DAQ Threshold Units (DTU)). The location and time stamp of the hit is stored 

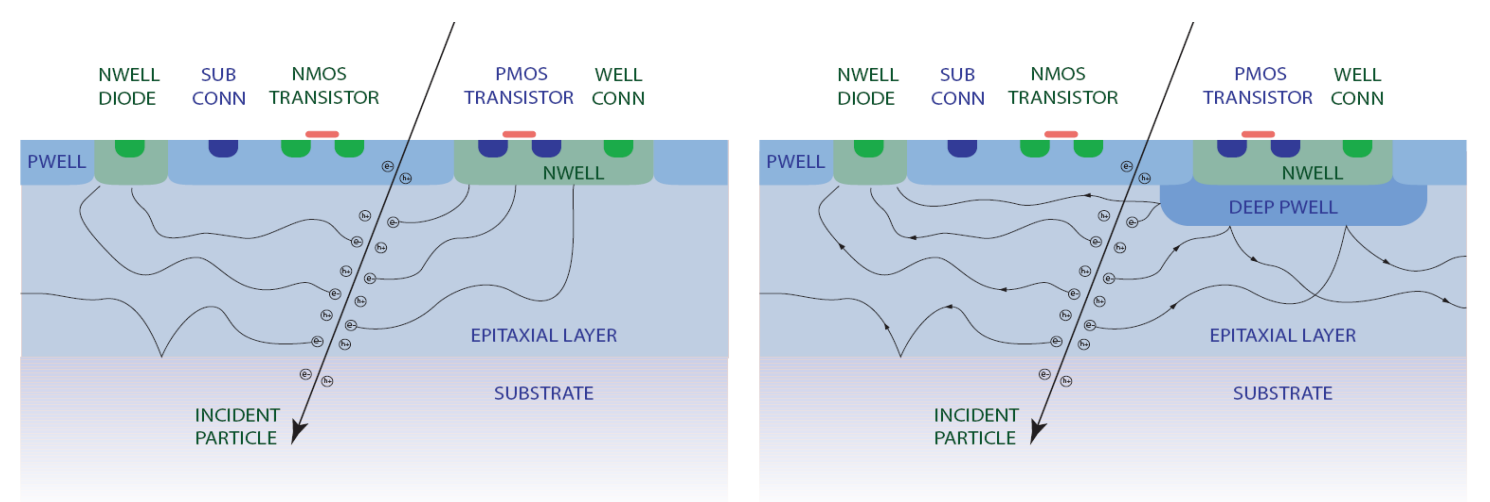

Figure 2. Schematic of a typical standard CMOS sensor demonstrating the charge diffusion to the diodes (left), and a CMOS sensor utilising INMAPS where the charge is repelled from the deep P-well (right).

and the complete sensor is read out at the end of each bunch train. Due to the size of the in-sensor memory, each segment can have a maximum of 19 hits in each bunch train before saturation occurs and it is thus essential to keep noise to a minimum to avoid losing genuine hits due to the memory filling.

\subsection{INMAPS process}

In a standard CMOS sensor, the charge diffuses to the signal diodes where it is collected. However, during diffusion, the charge is also collected parasitically at other $\mathrm{N}$-wells, reducing the collection efficiency. This causes a significant degradation in the sensor performance and prohibits the use of PMOS components. The INMAPS process has been developed [10] specifically to allow the $\mathrm{N}$-wells in the PMOS to be shielded using a deep P-well implant, as shown in figure 2. This deep P-well shields the parasitic N-wells of the PMOS components and as such decreases the parasitic charge collection. This allows full CMOS technology to be used whilst maintaining sensor performance. The INMAPS process also allows sensors to be fabricated with a high-resistivity substrate. There are four variants to the TPAC sensor:

- $12 \mu \mathrm{m}$ standard epitaxial layer,

- $12 \mu \mathrm{m}$ standard epitaxial layer with INMAPS (referred to as "standard" in this paper),

- $12 \mu \mathrm{m}$ high-resistivity epitaxial layer with INMAPS, and

- $18 \mu \mathrm{m}$ high-resistivity epitaxial layer with INMAPS.

\section{Sensor characterisation}

Each pixel is characterised by its pedestal and noise level. Both of these are found by performing a threshold scan on each pixel and looking at the number of hits as a function of the threshold. The threshold scans were conducted in a darkened box to ensure that there was no interference from photons causing genuine hits in the sensors. The results of such a scan for a typical pixel are 


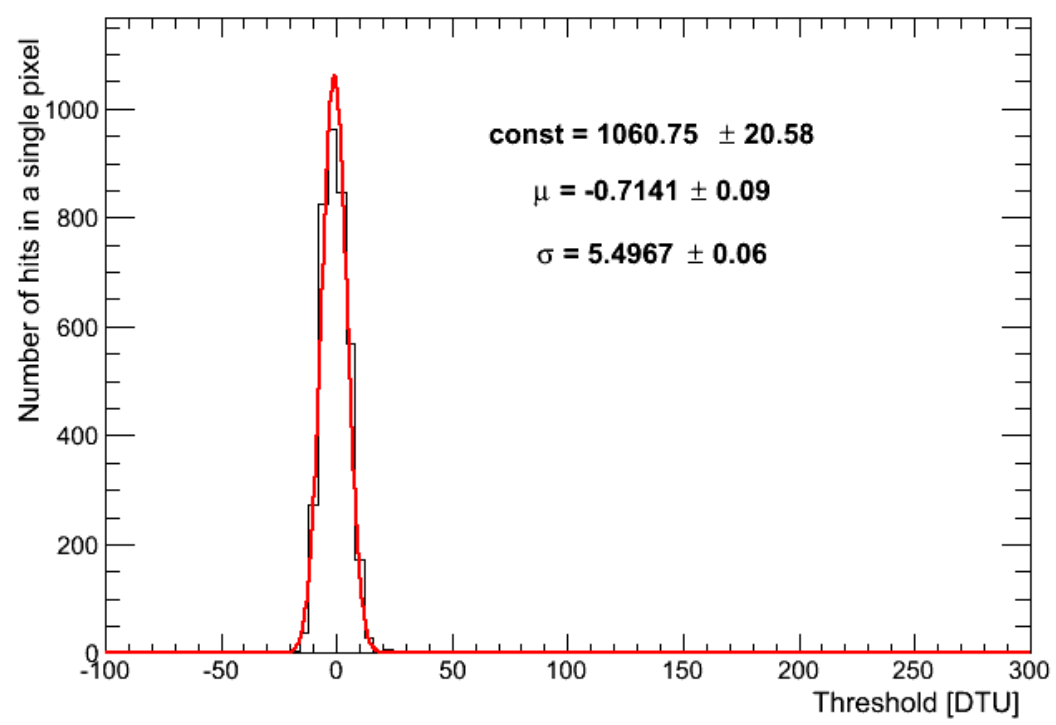

Figure 3. The results of a typical threshold scan on a single pixel where the mean is the pedestal and the width of the Gaussian is the noise in the pixel.
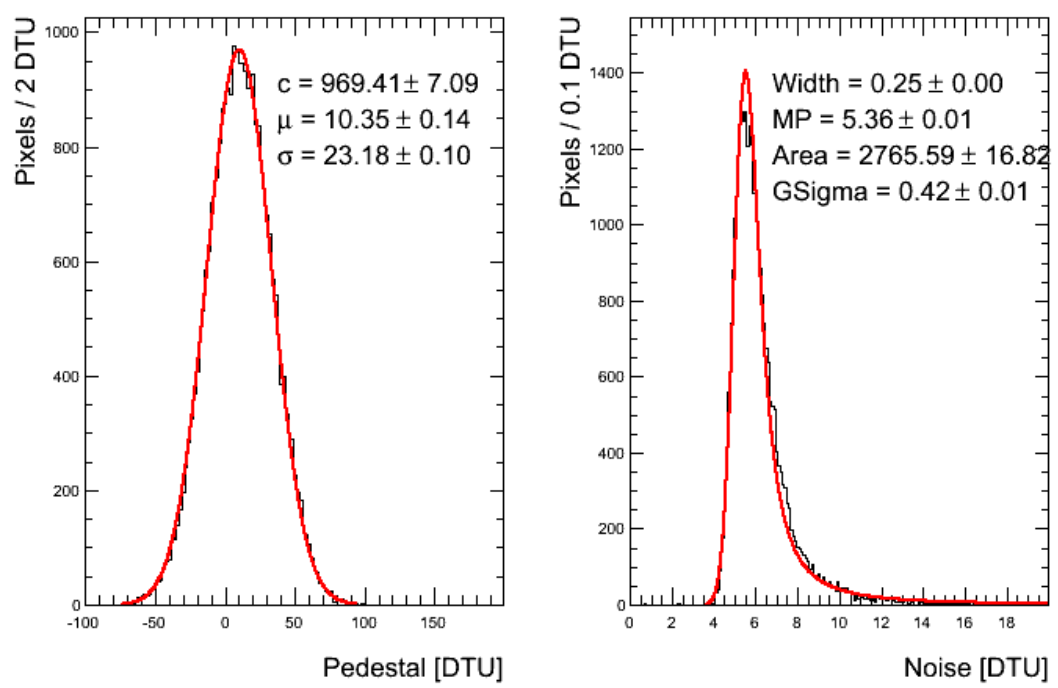

Figure 4. Example of the pedestal and noise spectra formed from scanning all 28224 pixels in a sensor with the fits overlaid.

shown in figure 3 where the mean value is the pedestal of the pixel and the width is the associated noise. Carrying out scans for all pixels in a sensor gives two corresponding spectra for each device (figure 4) which can be compared after each dose to investigate the changes to the sensor. The pedestal spectra were fitted with a simple Gaussian and the mean value extracted along with the corresponding error. The noise spectra were fitted using a Gaussian convoluted with a Landau distribution where the noise corresponds to the fit maxima.

During normal operation the sensors are trimmed so that all of the pedestals coincide at approximately 100 DTU. However, during the radiation tests the sensors were used untrimmed because the trimming process takes 60 hours per sensor and the changes in the noise and pedestal, rather 
than their absolute values, were the characteristics of interest. The relative changes to the noise were calculated using:

$$
f \Delta=\frac{X_{\text {Dose }}-X_{0 \mathrm{krad}}}{X_{0 \mathrm{krad}}},
$$

and the pedestal shift was calculated using:

$$
\Delta=X_{\text {Dose }}-X_{0 \mathrm{krad}}
$$

where $X_{\text {Dose }}$ and $X_{0 \mathrm{krad}}$ are the pedestal/noise value for a specific dose and zero dose respectively. The relative changes to the pedestal were not calculated as the pedestal of an untrimmed sensor is approximately zero leading to large relative values.

\section{Irradiation studies}

Eight sensors have been irradiated with $50 \mathrm{keV}$ x-rays, under two different bias, $0 \mathrm{~V}$ and $1.8 \mathrm{~V}$ (ground and operating voltages) up to doses of $5000 \mathrm{krad}$ and $200 \mathrm{krad}$ respectively, as summarised in table 1. Also studied were the effects of dose rate, with the sensors exposed at $60 \mathrm{rad} / \mathrm{s}$ and $6 \mathrm{rad} / \mathrm{s}$, and the effect of using a high resistivity epitaxial layer. The sensors held at ground were exposed in various dose steps of between $200 \mathrm{krad}$ and $1000 \mathrm{krad}$, while the sensors held at $1.8 \mathrm{~V}$ were exposed in steps of $20 \mathrm{krad}$. After each dose the pixels were characterised to quantify the effect on the pixels. The x-ray source delivered an exposure to all pixels that was uniform to within approximately $10 \%$ and therefore a relative uncertainty of $10 \%$ is assigned to the delivered dose.

The $\mathrm{x}$-rays will liberate electrons in the silicon with a maximum energy of $50 \mathrm{keV}$ which in turn will impart a maximum of $0.19 \mathrm{eV}$ upon a recoiled silicon atom. In silicon the displacement damage threshold is $21 \mathrm{eV} \mathrm{[16]} \mathrm{and} \mathrm{as} \mathrm{such} \mathrm{the} \mathrm{damage} \mathrm{to} \mathrm{the} \mathrm{sensors} \mathrm{cannot} \mathrm{be} \mathrm{from} \mathrm{bulk} \mathrm{damage}$ and must be due to surface effects such as charge trapping at boundary interfaces.

Between irradiations the sensors were kept in a freezer at $-20^{\circ} \mathrm{C}$ to limit the amount of annealing. As a threshold scan on all the pixels in the sensor took approximately 6 hours only a quarter of the sensor was scanned following each irradiation. This was essential as the annealing in the sensor would have been accelerated as the operating temperature of the sensors is greater than room temperature and this could have caused discrepancies in the results.

\subsection{Noise and pedestals - bulk pixels}

\subsubsection{Unpowered sensors}

Four standard sensors were held at ground during the exposure with two receiving integrated doses of $1000 \mathrm{krad}$ and the others receiving $5000 \mathrm{krad}$, all at a dose rate of $60 \mathrm{rad} / \mathrm{s}$. These studies were performed to allow a comparison between the effects of bias in the sensors during exposure (section 4.1.2).

At $1000 \mathrm{krad}$ the noise increased in all sensors between 5\% and 8\%. Pairs of sensors 3 and 6 , and sensors 1 and 4 were in good agreement within each pairing. At $5000 \mathrm{krad}$ the noise in sensor 3 had increased by $18 \%$ and sensor 6 by $21 \%$ (figure 5 ).

Over the same range of doses the pedestals also increased but by between $1.3 \mathrm{DTU}(10 \%)$ and $3.7 \mathrm{DTU}(25 \%)$ at $1000 \mathrm{krad}$ rising to a maximum increase of $3.9 \mathrm{DTU}(35 \%)$ at $5000 \mathrm{krad}$. As the 

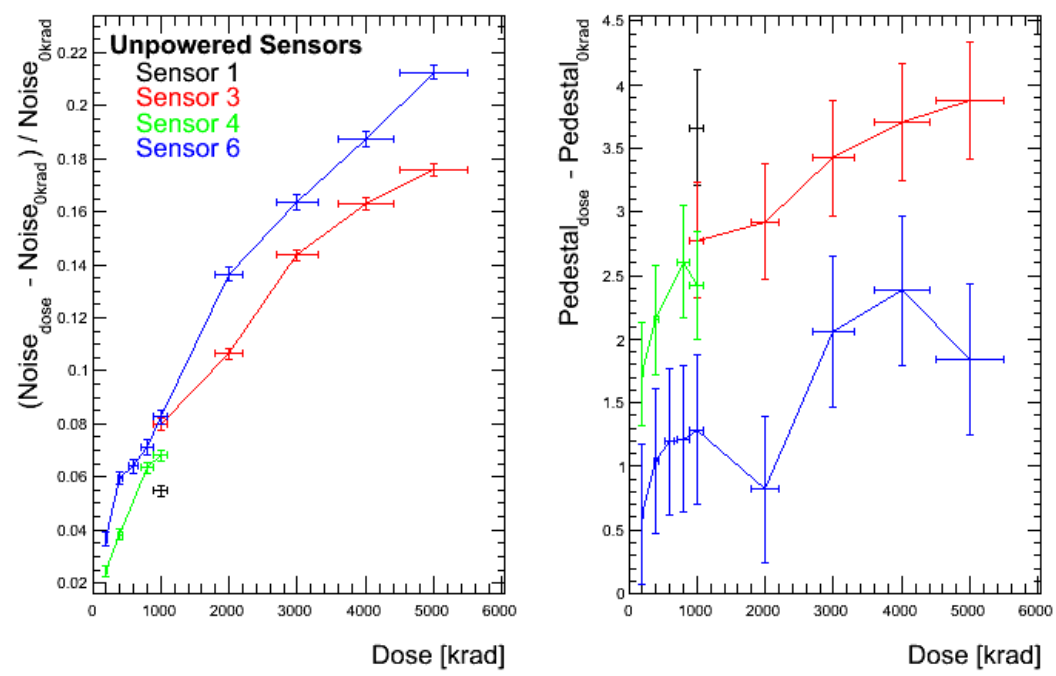

Figure 5. The fractional changes in noise (left) and absolute changes in pedestal (right) from zero dose for the four sensors held at ground during exposure.

changes in the noise and pedestals were different this suggests that there could be multiple sources of damage to the sensors caused by the radiation.

\subsubsection{Powered sensors}

Four additional sensors were exposed to radiation whilst held at their operating voltage of $1.8 \mathrm{~V}$. Two of these were standard sensors, and the third was a high resistivity $18 \mu \mathrm{m}$ sensor; all three were irradiated at a dose rate of $60 \mathrm{rad} / \mathrm{s}$. The fourth sensor was a standard sensor with a dose rate of $6 \mathrm{rad} / \mathrm{s}$ in order to test whether the results were rate dependent. Due to problems with loading configuration data on to the sensors (see section 4.1.3) the powered sensors were only exposed to small integrated doses of between $200 \mathrm{krad}$ and $240 \mathrm{krad}$ in stages of $20 \mathrm{krad}$.

Figure 6 shows that the noise once again increases with dose as expected with a maximum increase in sensor 9 of $9 \%$. The noise changes in the powered sensors at $200 \mathrm{krad}$ (4\% to $8 \%$ ) coincide with the noise changes in the unpowered sensors at $1000 \mathrm{krad}(5 \%$ to $8 \%$ ) implying that bias in the sensors accelerates the increase in the noise (see table 1). The noise in sensor 10 was observed to fall slightly. Although this sensor was exposed at the low dose rate it is believed that the decrease is more likely to be an artifact of the fit used to extract the noise.

The behaviour of the pedestal when the sensors were powered during irradiation was found to be very different to those that were held at ground. When powered the pedestal actually decreases with dose in all of the sensors up to $\sim 200 \mathrm{krad}$. A lower pedestal during sensor operation will lead to an increase in the noise rate of the sensors and as such this could be a very important feature at higher doses. The dose rate does not affect the pedestal shifts as the change in sensor 10 is in good agreement with the other sensors.

In the high resistivity epitaxial layer (sensor 12) the fractional changes in the noise are in agreement with the standard sensors. The pedestal actually decreases more than the standard sensors. This once again implies that there could be multiple sources for the damage to the sensor, an epitaxial layer independent source (noise) and an epitaxial layer dependant source (pedestal). 


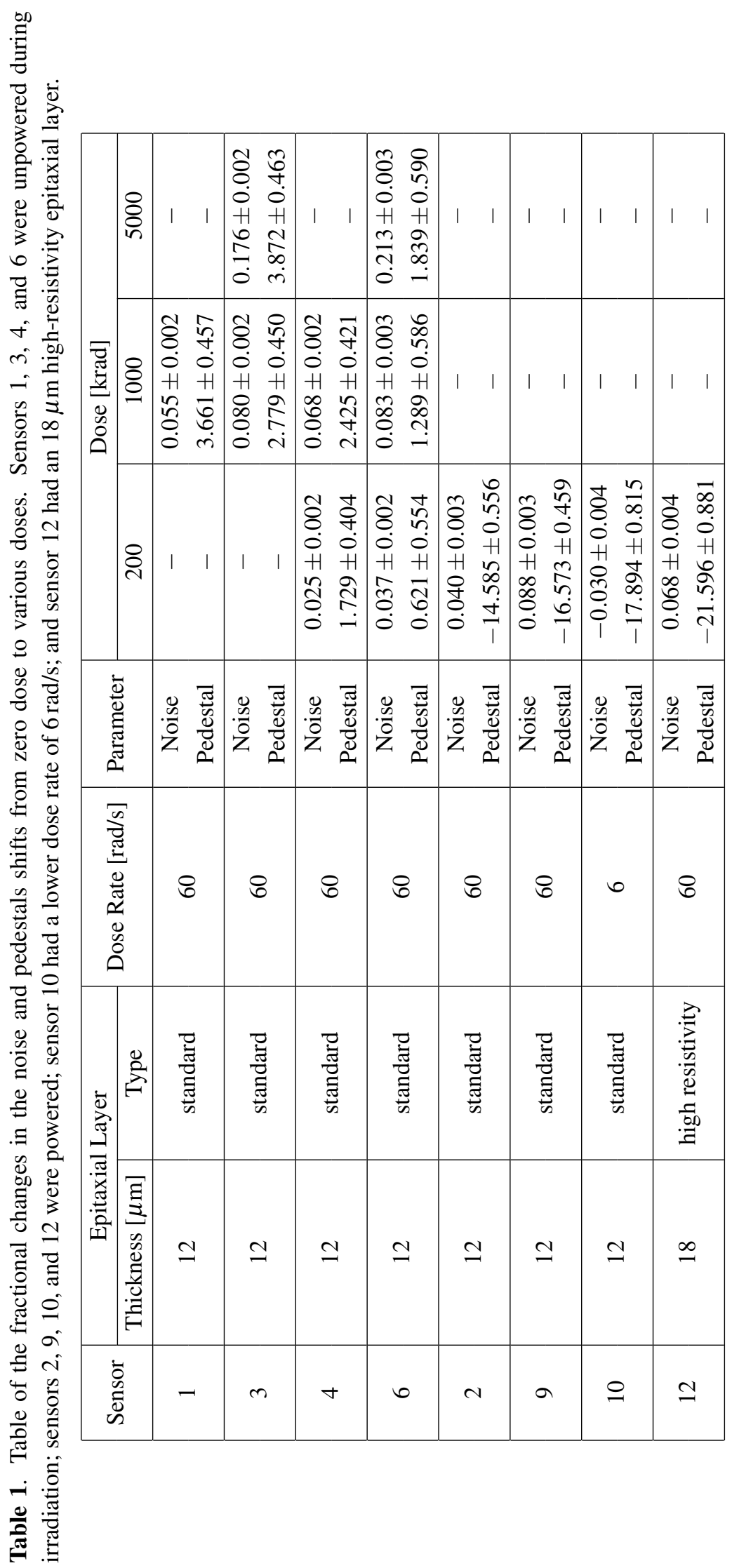



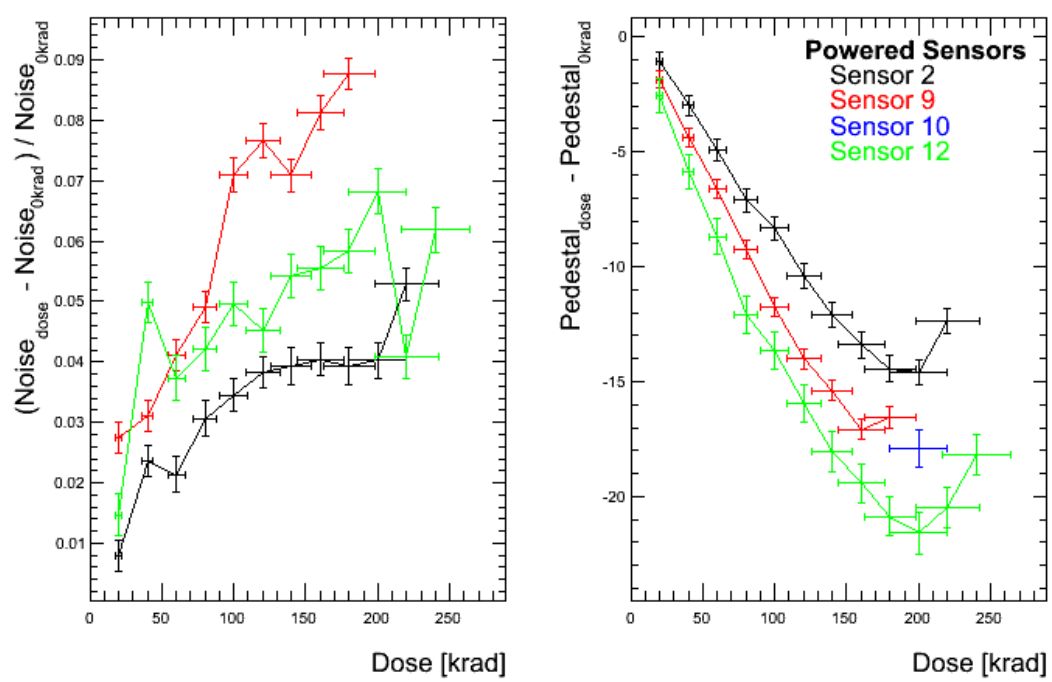

Figure 6. The fractional changes in noise (left) and and absolute changes in pedestal (right) from zero dose for the four sensors held at operating voltage. Sensor 10 was exposed at the lower rate of $6 \mathrm{rad} / \mathrm{s}$ and sensor 12 was the $18 \mu \mathrm{m}$ high resistivity epitaxial layer.

The pedestal changes actually decreased at the higher doses because problems with the shift register meant that the sensors underwent annealing whilst waiting for the sensors to be scanned. This set the limit on the maximum dose for which reliable characterisation of the sensors could be performed, as described below.

\subsubsection{Shift register problems}

The configuration data is loaded on to the pixels by a three-phase clock shift register and this data contains the trim values of each pixel as well as the pixel masks, which can be used to define a set of pixels to be excluded from the readout. In the TPAC sensor, if more than a few hundred pixels fire at the same time, noise pickup is observed in the pixels [14]. To work around this problem, during a threshold scan only one column of 168 pixels was unmasked at a given time. At the higher doses the values read in to and subsequently returned from the shift register were different in some pixels. Due to the nature of the shift register structure, a single bad register in a column of pixels can corrupt the whole column. This leads to the possibility of the mask values in an entire column being invalid and unmasking an additional 168 pixels during the scan. If there are too many bad columns it can prevent the sensor being reliably read out until the shift register is repaired. There are two potential ways of repairing the shift register; adjusting the voltage that drives the shift register; or allowing the sensor to anneal.

Figure 7 shows the number of bad columns in the shift register for various shift register supply voltages at doses of $0,100,200$, and $220 \mathrm{krad}$ for sensor 2 . This sensor had one bad column in the shift register before exposure so it was essential not to develop any more. It is clear that at $100 \mathrm{krad}$ there is a large range of voltages over which just one bad column is present in the register, so the sensor functions correctly. At $200 \mathrm{krad}$ this behaviour has changed so that there is no voltage at which the register has just one bad column and with a further increase in dose up to $220 \mathrm{krad}$ the 


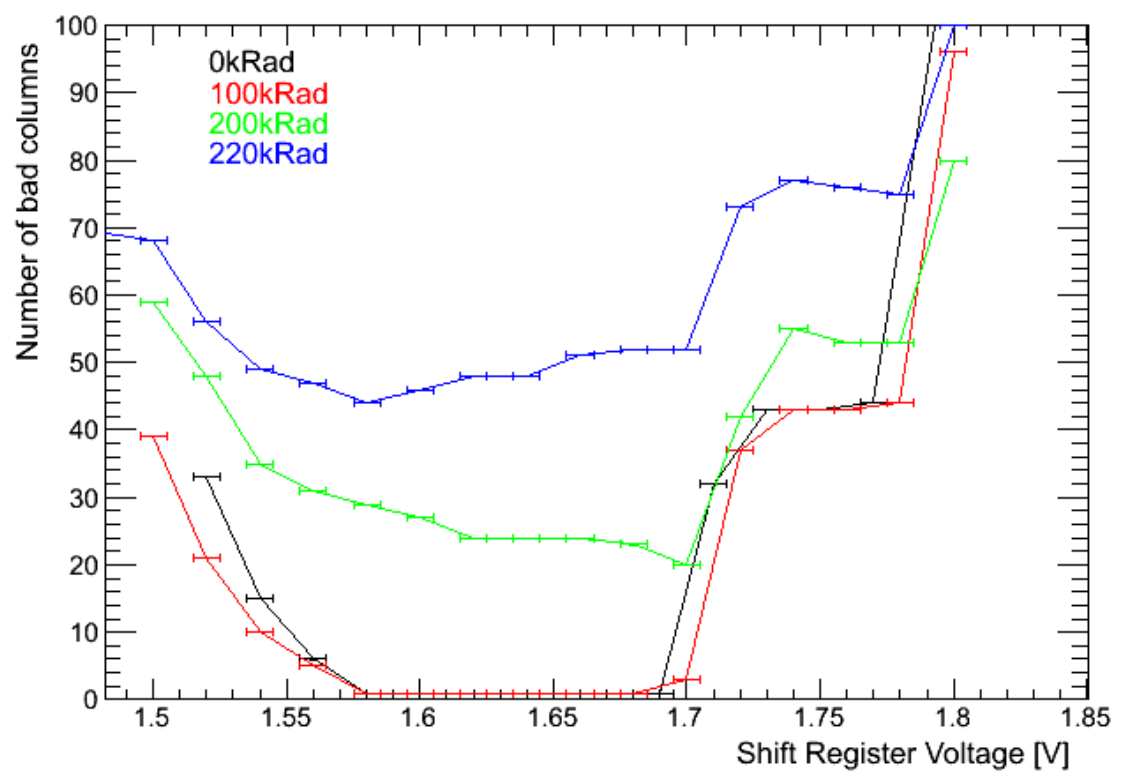

Figure 7. The number of bad columns in the shift register as a function of supply voltage for various doses of a standard $12 \mu \mathrm{m}$ sensor.
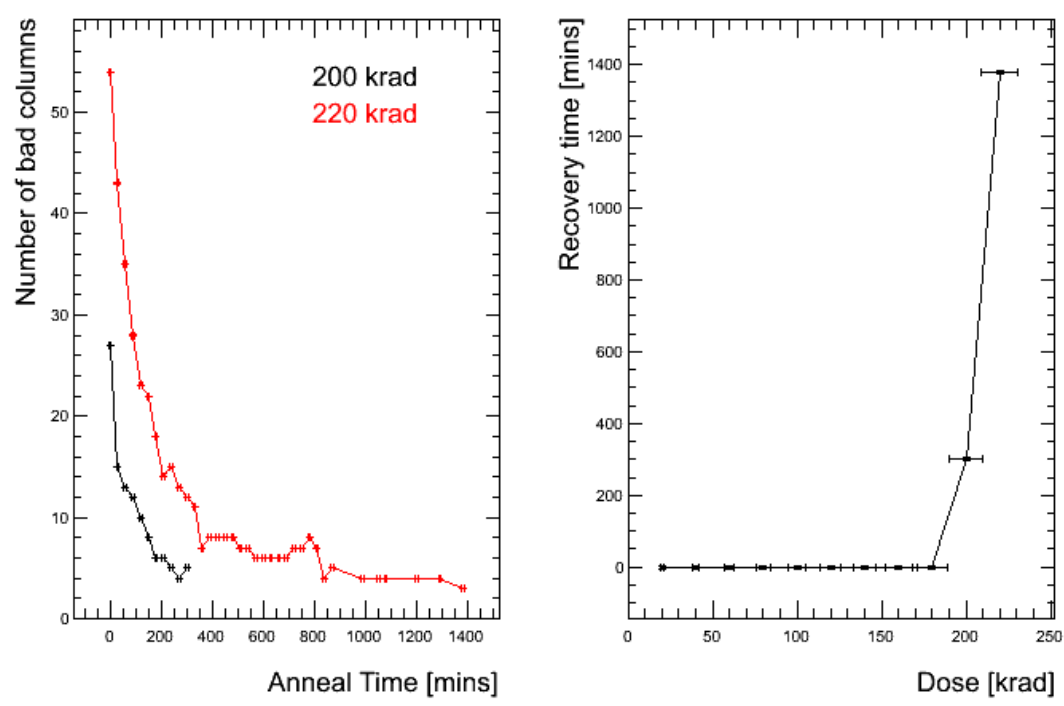

Figure 8. The number of bad columns vs anneal time for two different doses (left) and the total anneal time before the sensor could be reliably scanned vs dose for the same sensor (right).

number of bad columns has significantly increased. At these higher doses, adjusting the supply voltage does not solve the issue and as such the register must be allowed to anneal.

With the shift register supply voltage fixed at the design voltage of $1.65 \mathrm{~V}$, the behaviour was studied in 30 minute intervals until the number of bad columns was sufficiently small that the sensor could be reliably read out. Figure 8 reiterates the increased number of bad columns with a higher dose and shows the progression of the recovery of the shift register. The time to recover increased from 300 minutes to 1400 minutes for 200 and $220 \mathrm{krad}$ respectively. Because these large waiting 


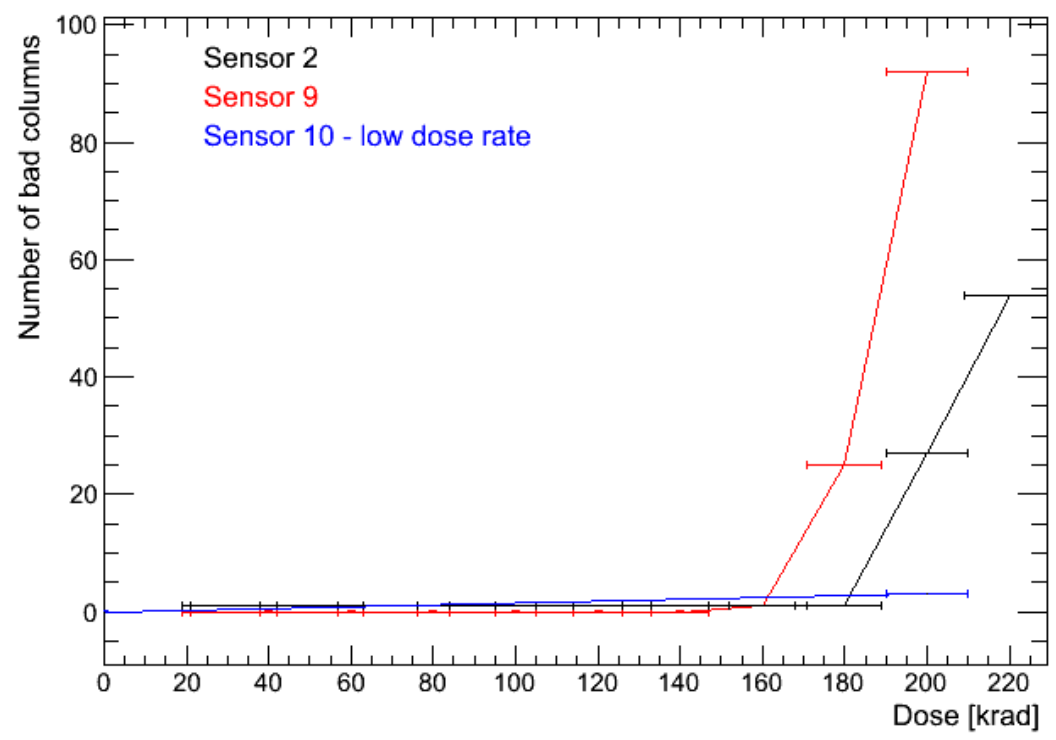

Figure 9. The number of bad columns vs dose for the two standard sensors exposed at a rate of $60 \mathrm{rad} / \mathrm{s}$ and the standard sensor at $6 \mathrm{rad} / \mathrm{s}$ (sensor 10$)$.

times allow the damage to the sensor to anneal away, it was not possible to carry out measurements at the larger doses.

When the dose rate was reduced by a factor of 10 , the shift register problems were vastly reduced. At $200 \mathrm{krad}$ there were just three bad columns in the sensor exposed at the reduced rate compared to more than 20 for the other two standard sensors (see figure 9). This suggests that the shift register is annealing during exposure. When the sensors are held at ground during exposure the shift register behaves as expected up to very high doses so the bias in the sensor is impeding the recombination of the electron-hole pairs created by the radiation. As the pedestal shift in the low dose rate sensor is equivalent to the shift in the other sensors this implies that the damage to the shift registers is rate dependent and the damage causing the pedestal changes is rate independent. Due to this, in the low radiation level environments of a calorimeter system the sensor should be able to withstand integrated doses higher than those tested as the dose rate will be significantly lower.

\subsection{Signal-analogue test pixels}

The sensors were designed with two "test" pixels having analogue readout to complement the digital pixels and to allow the shape and the characteristics of the signal in the pixels to be studied. An Fe-55 source was placed adjacent to the test pixels, a threshold of $140 \mathrm{mV}$ was set and any signal in the pixels greater than this threshold had the signal shape measured and recorded using an oscilloscope. The minima of the signal was found by fitting a second order polynomial and an energy spectrum was built up for the pixels' response (see figure 10).

$\mathrm{Fe}-55$ produces gamma radiation with two characteristic photons, $\mathrm{k}_{\alpha}$ and $\mathrm{k}_{\beta}$, having energies of $5.9 \mathrm{keV}$ and $6.5 \mathrm{keV}$ respectively. The $\mathrm{k}_{\alpha}$ peak is the dominant decay with the $\mathrm{k}_{\beta}$ having a reduced rate. When the photons deposit energy in $12 \mu \mathrm{m}$ silicon they will liberate $1620 e^{-}$and $1778 e^{-}$respectively. When one of the photons from the decay interacts directly with one of the 

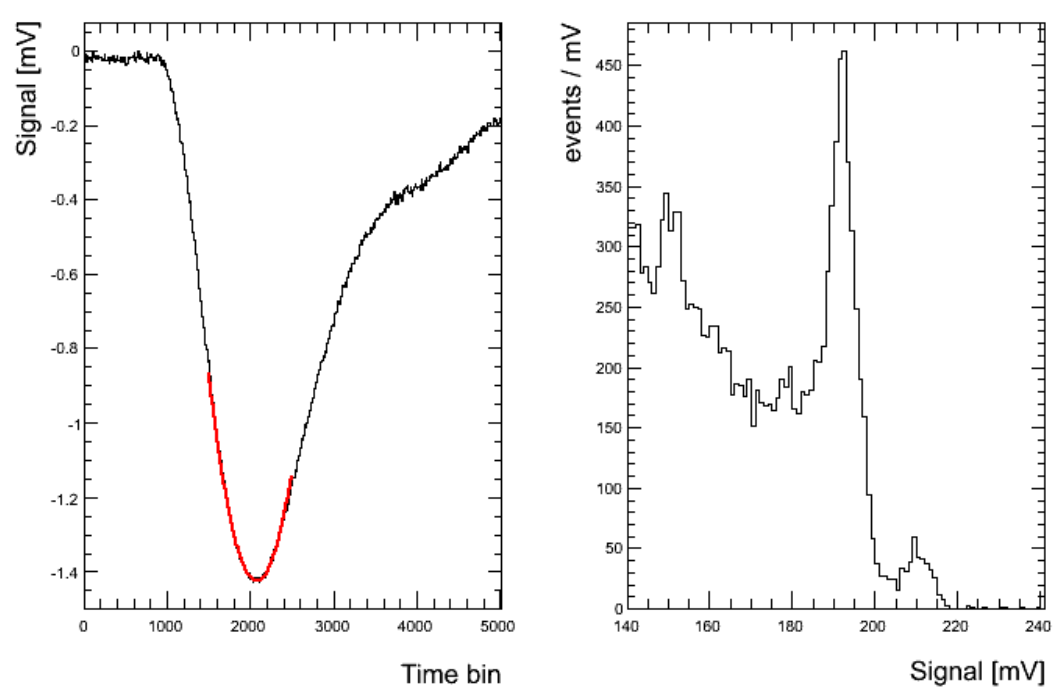

Figure 10. An example of the oscilloscope trace of an Fe-55 event with fit overlaid (left), and the resulting spectrum created using the minima of each event (right).

diodes in the sensor, all of the energy is collected and the full characteristic energy peaks are observed in the spectrum. If the photon interacts in the epitaxial layer outside of the diodes, charge diffusion leads to charge being lost into neighbouring pixels. The amount of charge lost is dependent on the location of the initial photon and therefore there are many events which see a charge collection below the characteristic peaks [13]. The gain of the sensors can be calculated using the $\mathrm{k}_{\alpha}$ peak, where all $1620 e^{-}$are collected by

$$
\text { Gain }=\frac{\text { Signal }[\mathrm{mV}]}{1620 e^{-}},
$$

where Signal is the mean value of a Gaussian fit to the $\mathrm{k}_{\alpha}$ peak.

Four sensors were tested before and after being irradiated and annealing had occurred. To collect a sufficient number of events to resolved the $\mathrm{k}_{\alpha}$ peak a single Fe-55 scan took approximately 24 hours. For this reason the signal could only be studied after a long period of annealing to ensure that the results were not affected by annealing. Three of the sensors were powered during the exposure and the fourth was held at ground and received a much higher integrated dose. Prior to the sensors being exposed, the $\mathrm{k}_{\alpha}$ peak positions agreed within an acceptable spread of $10.8 \mathrm{mV}$. The $\mathrm{k}_{\alpha}$ peak in sensor 12 has an increased width due to the additional charge collection adjacent to the sensor diodes in the high resistivity silicon. Post exposure, the position of the $\mathrm{k}_{\alpha}$ peak increased in all of the sensors (see figure 11) with a final spread of $9.4 \mathrm{mV}$.

\subsection{Signal to noise}

Table 2 gives a summary of the signal and gain for the test pixels obtained with the Fe- 55 scans, and the noise of the bulk pixels from the threshold scans in DTU. Although the observed signal and gain increased with radiation, the critical quantity is the signal to noise ratio $(\mathrm{S} / \mathrm{N})$. The fractional change was calculated using equation (3.1) whilst $\mathrm{S} / \mathrm{N}$ was given by:

$$
\frac{\mathrm{S}}{\mathrm{N}}=\frac{\text { Signal }[\mathrm{mV}]}{\text { Noise }[\mathrm{mV}]}
$$



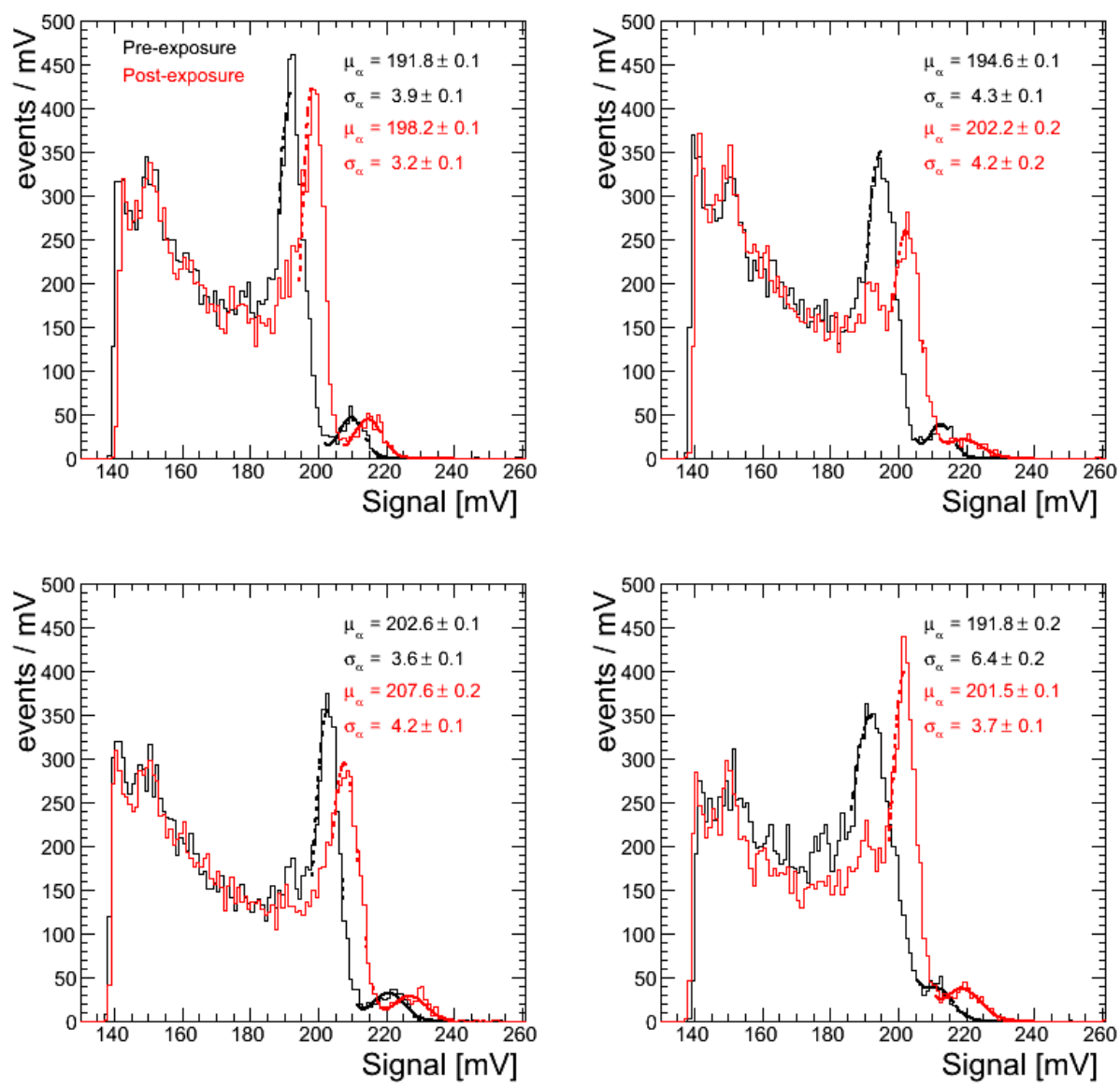

Figure 11. The charge collection spectra for all sensors before and after irradiation and annealing where black is pre and red post exposure for sensors 2, 6 (unpowered), 9, and 12 (high resistivity).

where Signal is the position of the $\mathrm{k}_{\alpha}$ centroid and Noise is the noise taken from the threshold scans (in DTU) converted into $\mathrm{mV}$ by

$$
\text { Noise }[\mathrm{mV}]=\text { Noise }[\mathrm{DTU}] \times C\left[\mathrm{DTU} / e^{-}\right] \times \text {Gain }\left[\mathrm{mV} / e^{-}\right] \text {, }
$$

where $C$ is the conversion factor of DTU to electrons (3.3 DTU/ $e^{-}[14]$ ), and Gain is calculated using equation (4.1). The values of the noise in electrons and $\mathrm{mV}$ are also given in table 2 .

Across all four sensors, $\mathrm{S} / \mathrm{N}$ was observed to decrease after irradiation. When the sensor was powered during irradiation $\mathrm{S} / \mathrm{N}$ decreased by between $4 \%$ and $8 \%$. The results for the high resistivity sensor $(6 \pm 1 \%)$ were consistent with the two standard sensors $(4 \pm 1 \%$ and $8 \pm 1 \%)$ demonstrating that the changes in $\mathrm{S} / \mathrm{N}$ are independent of the epitaxial layer. The sensor irradiated at ground up to $5000 \mathrm{krad}$ underwent a $\mathrm{S} / \mathrm{N}$ decrease of $15 \pm 1 \%$ showing that the sensor is capable of receiving large doses with a relatively small effect on $\mathrm{S} / \mathrm{N}$. 


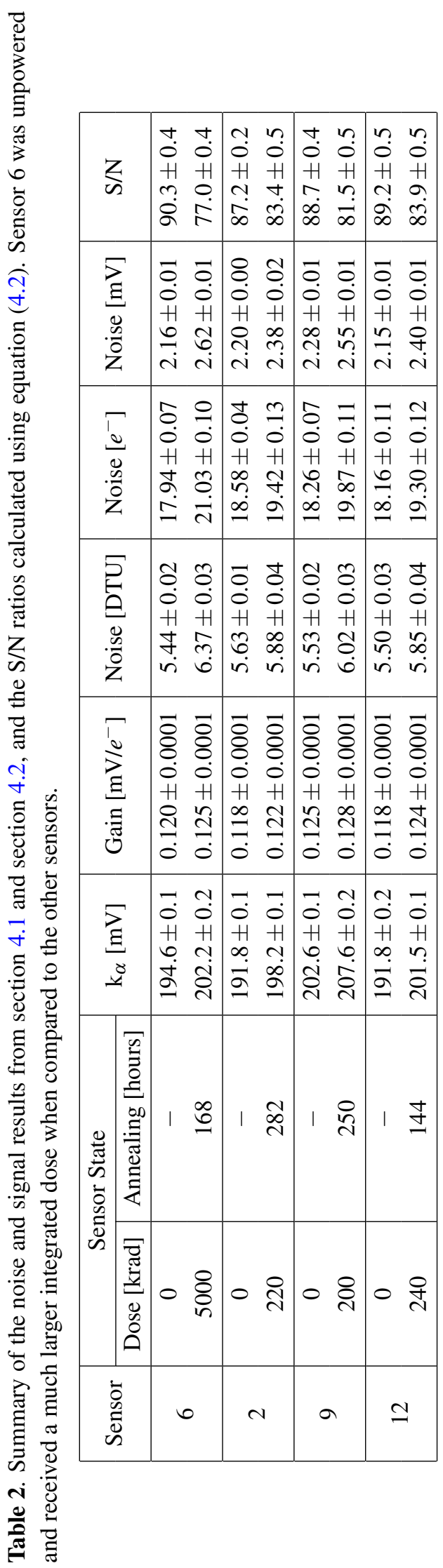




\section{Conclusions}

It has been shown that the noise in the TPAC sensors has increased by between $2.5 \%$ and $8 \%$ at $200 \mathrm{krad}$ for various sensor bias rising to $21 \%$ at $5000 \mathrm{krad}$ for a sensor held at ground during exposure. The $\mathrm{S} / \mathrm{N}$ has been shown to fall by a maximum of $8 \%$ in sensors at a dose of $200 \mathrm{krad}$. At $5000 \mathrm{krad}$, the $\mathrm{S} / \mathrm{N}$ has reduced by just $15 \%$ which is very promising for future applications.

It has been demonstrated that changes in $\mathrm{S} / \mathrm{N}$ are independent of epitaxial layer type as the sensors irradiated whilst powered were all in agreement. When the sensors are irradiated whilst powered, problems develop in the shift register leading to the need for a redesign of the shift register in future iterations of the sensors. The reduced dose rate gave rise to a vastly reduced number of configuration errors in the shift register. A dose rate of $6 \mathrm{rad} / \mathrm{s}$ yielded just three bad columns compared to greater than 20 bad columns for a dose rate of $60 \mathrm{rad} / \mathrm{s}$. The dose rate received by the Silicon Inner Tracker, Silicon External Tracker, and the ECAL in CLIC ILD, and the ECAL in ILC ILD is orders of magnitude lower than $6 \mathrm{rad} / \mathrm{s}$ and as such the shift register issues would not present a problem even using the current TPAC technology.

\section{Acknowledgments}

We would like to thank Matt Wilson of Rutherford Appleton Laboratory and STFC for help and support during the course of sensor irradiations.

\section{References}

[1] R. Turchetta, CMOS sensors for the detection of minimum ionising particles, in Proceedings of the IEEE Workshop on Charge-Coupled Devices and Image Sensors, Lake Tahoe U.S.A. (2001), pp. 7-9.

[2] E.R. Fossum, CMOS image sensors: electronic camera-on-a-chip, IEEE Trans. Electron Dev. 44 (1997) 1689.

[3] R. Turchetta et al., A monolithic active pixel sensor for charged particle tracking and imaging using standard VLSI CMOS technology, Nucl. Instrum. Meth. A 458 (2001) 677.

[4] CALICE-UK Home Page, http://www.hep.ph.ic.ac.uk/calice/.

[5] CliC Physics Working Group collaboration, E. Accomando et al., Physics at the CLIC multi-TeV linear collider, hep-ph/0412251.

[6] Compact Linear Collider, http://clic-study.org/.

[7] A.R. Faruqi, R. Henderson and J. Holmes, Radiation damage studies on STAR250 CMOS sensor at $300 \mathrm{keV}$ for electron microscopy, Nucl. Instrum. Meth. 565 (2006) 139.

[8] B. Dryer et al., Gamma radiation damage study of $0.18 \mu \mathrm{m}$ process CMOS image sensors, Proc. SPIE 7742 (2010) 77420E.

[9] M. Esposito, T. Anaxagoras, O. Diaz, K. Wells and N.M. Allinson, Radiation hardness of a large area CMOS Active Pixel Sensor for bio-medical applications, ISDI White Paper (2012).

[10] J.A. Ballin et al., Monolithic Active Pixel Sensors (MAPS) in a quadruple well technology for nearly 100\% fill factor and full CMOS pixels, Sensors 8 (2008) 5336 [arXiv : 0807.2920]. 
[11] A. Bevan et al., Design study for a next generation B factory pixel vertex detector, Nucl. Instrum. Meth. A 643 (2011) 29.

[12] L. Musa, Conceptual design report for the upgrade of the ALICE ITS, Technical Report CERN-LHCC-2012-005, LHCC-G-159 (2012).

[13] J.A. Ballin et al., Design and performance of a CMOS study sensor for a binary readout electromagnetic calorimeter, 2011 JINST 6 P05009.

[14] SPIDER collaboration, P. Dauncey, Performance of CMOS sensors for a digital electromagnetic calorimeter, PoS (ICHEP 2010) 502.

[15] SPIDER collaboration, DESY PRC Report (2009), http://www.hep.ph.imperial.ac.uk/calice/official/091105prc/spider.pdf.

[16] A. Akkerman et al., Updated NIEL calculations for estimating the damage induced by particles and $\gamma$-rays in Si and GaAs, Radiat. Phys. Chem. 62 (2001) 301. 